\title{
LA INVESTIGACIÓN FORMATIVA EN COLOMBIA: UNA MIRADA DESDE SU IMPLEMENTACIÓN
}

\section{FORMATIVE RESEARCH IN COLOMBIA: A LOOK FROM ITS IMPLEMENTATION}

\author{
Juan Carlos Cruz Ardila ${ }^{2}$ \\ Jairo Alberto Olarte Cabana ${ }^{3}$ \\ Sergio Hernández Vásquez ${ }^{4}$ \\ Edwin Arbey Hernández García ${ }^{5}$
}

\section{RESUMEN}

Apartedelasactividades vinculadasaladocencia, la investigación formativa es otro de los grandes ejes asociados a la educación superior a nivel global: semilleros de investigación, trabajos de grado, proyectos de aula, entre otros aspectos son relevantes para pensar este aspecto que $1 \quad$ Este trabajo hace parte del proyecto de investigación "Investigación formativa. Un análisis fundamentado en las experiencias de la Mesa Sur Pacífico de Investigación de ACIET" aprobado en la convocatoria interna de Unicamacho con el código Pl-0121 con resolución 425 del 9 de noviembre de 2021.

2 Ingeniero Electricista. Magister en Ingeniería. Grupo Inteligo. jcarloscruz@admon.uniajc.edu.co 00000001-6699-4149

3 Contador público. Magister en contabilidad. Grupo de Estudios en Gestión Empresarial. jalbertoolarte@ ucompensar.edu.co 0000-0002-9584-6599

4 Sociólogo. Master en Sociología y antropología de la Universidad Paris Diderot. Grupo de investigación Communitas Sinergia. Director de investigaciones en CUDES. investigaciones@cudes.edu.co 0000-0001-8187-628X 5 Economista. Magíster en Economía Aplicada. Grupo de Investigación Communitas Sinergia. edwinarbeyh@gmail.com 0000-0002-5919-7659 marca el devenir universitario. En este sentido, este documento tiene por objetivo generar una reflexión acerca de la forma y contenido como se han implementado los procesos de investigación formativa en Colombia. Para alcanzarlo, se parte de un análisis documental, en el que se analiza la producción científica acerca del problema aquí esbozado para después plantear algunas reflexiones sobre este proceso en las Instituciones de Educación Superior (IES) colombianas. En su estructura, este artículo está dividido en tres bloques: EI primero se dedica a establecer un panorama de la investigación en Colombia a partir de datos sociales y económicos. El segundo pretende establecer cuáles son aquellos aspectos de investigación asociados a las IES en Colombia; como consecuencia, las categorías asociadas a las líneas de investigación, estatus del profesor, inversión en investigación, entre otros aspectos 
son constitutivos de este apartado. Finalmente, las reflexiones acerca de la investigación formativa aplicada a Colombia se presentan a manera de conclusiones, donde se destaca la manera difusa con la que se define este tipo de investigación en Colombia, la necesidad de un nuevo planteamiento del uso de recursos y métodos por parte de los profesores y lo cerrado que pueden llegar a ser los espacios de investigación formativa en las IES.

PALABRAS CLAVE: Investigación, trabajo colaborativo, formación en investigación, educación, semilleros de investigación, práctica educativa.

\section{ABSTRACT}

Apart from the activities related to teaching, formative research is another of the main axes associated with higher education al global level: research seedbeds, degree projects, classroom projects, among other aspects are relevant to think about this aspect that marks becoming a university. In this sense, this document aims to generate a reflection on the form and content of how formative research processes have been implemented in Colombia. To achieve this, we start from a documentary analysis, in which the scientific production about the problem outlined here is analyzed and then some reflections on this process in Colombian Higher Education Institutions (HEI) are raised. In its structure, this article is divided into three blocks: The first is dedicated to establishing an overview of research in Colombia based on social and economic data; the second, will establish what are those research asspects associated with HEI in Colombia; as a consequence, the categories associated with the lines of research, status of the professor, investment in research, among other aspects are considered in this section. Finally, the reflections on formative research applied to Colombia are presented in a way of conclusions, wich highlights the diffuse way like this type of research is defined in Colombia, the need for a new approach to use of resources and methods by part of the professors and how closed the formative research spaces in $\mathrm{HEI}$ can become.

KEYWORDS: Research, collaborative work, research training, education, educational practice, seedbeds of research

\section{INTRODUCCIÓN}

Los países de América Latina atraviesan acelerados procesos de cambio, cuyas repercusiones se han percibido tanto en el ámbito político, como en el económico y social. Hoy se verifican avances extraordinarios en términos de capacidad tecnológica, científica y productiva, pero al mismo tiempo se evidencian desigualdades profundas en los niveles de desarrollo en la región. Un contexto internacional, marcado por lo que se ha denominado la globalización, ha generado simultáneamente grandes oportunidades y profundas dificultades para las poblaciones latinoamericanas (Serbin, 2018).

En este contexto, existe un amplio y creciente consenso acerca de que el futuro de las sociedades humanas dependerá, en gran parte, de su capacidad para asegurar la creación de nuevo conocimiento (Correa D., Benjumea A., \& Valencia A., 2019). La investigación científica y el desarrollo tecnológico, así como la capacidad para adaptar y adoptar nuevas tecnologías, constituyen hoy por hoy un elemento estratégico para aumentar la competitividad en la economía mundial y para lograr el bienestar colectivo.

Así mismo, del consenso se extrae la tesis que las Instituciones de Educación Superior (IES), centros de investigación por excelencia, se enfrentan al reto de ayudar a sus países en la estimulación de la economía y en asegurar 
un proceso equitativo de desarrollo sostenible (UNESCO -IESALC y Universidad Nacional de Cordoba, 2018). Sin embargo, es esencial reconocer que a menos que ellas mismas participen en un proceso de transformación global, y que la sociedad y el Estado apoyen esos cambios, su capacidad para convertirse en actores claves del desarrollo social y económico será muy limitada.

De otra parte, las IES enfrentan el reto de incrementar el capital humano en sus docentes y estudiantes, especialmente lo relacionado con la investigación en su sentido más amplio; esto, por supuesto conlleva procesos de inversión en recursos financieros, tecnológicos, humanos, entre otros, importantes si la meta es crecer en productos resultados de investigación. En este sentido y recogiendo las ideas generales expresadas en los párrafos anteriores, se puede decir que la investigación presenta desafíos tanto externos como internos a las IES, de donde los factores internos, especialmente los relacionados con la investigación formativa están a la orden del día.

Por lo cual, el objetivo de este artículo es reflexionar acerca de la forma y fondo como se han venido implementando los procesos de la investigación formativa en Colombia, tales que conduzca a la mejora continua de dichos procesos desde el punto macro como país y desde el punto micro para las Instituciones de Educación Superior (IES) en Colombia.

Metodológicamente, primero se hará una mirada a los hechos y datos sociales y económicos de tipo macro sobre la investigación en Colombia, luego reflexiones sobre la investigación formativa, como caso particular, en las IES, para terminar el artículo con reflexiones propias de los autores acerca de la implementación de la investigación formativa en las IES colombianas.

\section{HECHOS Y DATOS MACRO DE LA INVESTIGACIÓN EN COLOMBIA}

Actualmente se está atravesando una época de grandes decisiones donde la producción, el tratamiento y la circulación de la información son condiciones para el desarrollo tecnológico y la innovación, cuando se establece una economía del saber con campos especializados y nuevos derechos, tal como la propiedad intelectual, que establece nuevas reglas de repartición de la riqueza de las naciones. El futuro requiere de una conciencia y de una voluntad colectivas para tomar medidas decisivas. La inversión masiva en los cerebros, la renovación de las Universidades como lugar de desarrollo y valorización de los recursos humanos, son condiciones para enfrentar cambios estructurales y duraderos. La importancia de los desafíos justifica que, no solo los gobiernos, sino también los universitarios y las sociedades civiles, decidan sobre el destino de su nación.

En Colombia, el diagnóstico del estado del sistema de enseñanza superior fue hecho en varias oportunidades por las autoridades gubernamentales. Uno de los rasgos característicos es el que llaman la atomización institucional del sistema, que resultó agudizada por el crecimiento de la enseñanza privada, fenómeno que también ha afectado al Sistema de Ciencia y Tecnología, en el que el sector privado es parte de los organismos rectores. El Banco Mundial (2018) manifiesta que un sistema educativo incluyente fomenta la libertad individual y el bienestar social, igualmente debe fomentar el empleo, incrementar el ingreso, mejorar la salud y reducir la pobreza. Esto conlleva a un fortalecimiento de las instituciones y una promoción de la cohesión social (p.15)

Así mismo, el reto de la investigación es cada día más difícil, por cuanto la capacidad para generar, apropiarse y aplicar conocimientos y tecnologías tiene una diferencia sustancial 
respecto de la sociedad del conocimiento global, aumentando las distancias enormes que separan a la región de los países con mayor desarrollo. Todavía se evidencia baja inversión en Ciencia y Tecnología, estimada, en términos de porcentaje del Producto Interno Bruto, en un tercio de lo que destinan los países desarrollados, siendo para el 2018 de $0.61 \%$ frente al $2 \%$ de los países que hacen parte de la OCDE (Pardo, 2019).

Otra razón que ha condicionado la mejora de la investigación en Colombia, ha sido especialmente el hecho de que los sistemas de educación superior han evolucionado independientemente de los sistemas nacionales de ciencia y tecnología. Los organismos nacionales de ciencia y tecnología mantuvieron las tradicionales políticas gubernamentales de corto plazo, sin lograr introducir en las agendas políticas la coordinación de la formación de recursos humanos de alto nivel, la actualización permanente de la infraestructura para la investigación científica, el estímulo a la creación de grupos de trabajo y la generación de masas críticas de investigadores en determinados temas prioritarios.

Sin embargo, desde el punto de vista institucional, el Gobierno Nacional proyectó que con la creación del Ministerio de Ciencia, Tecnología e innovación (MINCIENCIAS) se pudiera incrementar las inversiones en actividades de ciencia, tecnología e innovación (ACTI) en 1,5\% como porcentaje del producto interno bruto (PIB) y analizar cuál es el rol y el aporte de la CTI desde la política pública para resolver y fortalecer los problemas de la sociedad basada en el conocimiento (Pardo, 2019, p.1), la cuestión hoy día es que con la pandemia del COVID19 dichas proyecciones se ponen en entredicho por la carga al déficit fiscal gubernamental (Comisión Económica para América Latina y el Caribe (CEPAL), 2020).
Por otra parte, es posible identificar otras limitantes en las IES que afectan los sistemas de investigación. Ellas son: Universidades en las que existen pocas acciones de investigación y falta de estrategias nuevas para la generalización de la investigación tanto formativa como científica. $Y$ también el número insuficiente de investigadores reconocidos por MINCIENCIAS, lo cual se refleja en las publicaciones $u$ otros modos de socialización.

En este último sentido, Rodríguez y Torres (2020) expresan que los profesores con doctorado están asumiendo responsabilidades de docencia adicionales y administrativas, donde mencionan que hay un crecimiento en los profesionales graduados como doctores, los cuales deben generar un impacto en el desarrollo social y también en el sistema educativo en el que se encuentran inmerso ( $p .46)$

Más que otros países, Colombia experimenta una laguna en la mayoría de las Universidades con respecto a las actividades de investigación. Por un lado, los estudios de posgrado, especialmente los de nivel doctoral, apenas se están posicionando. Por otro lado, con un nivel de escolaridad promedio que es bajo, los profesores no representan una masa crítica suficiente para realizar actividades de investigación de calidad. Por esta razón Martínez y Beleño (2020, p, 263) manifiestan que los docentes deben concentrar su ejercicio en enseñar para la vida y no para el momento, lo cual los obliga a adquirir elementos que respondan a las tendencias del mundo de hoy, convirtiéndose la situación en un problema centrado en la formación docente, de tal forma que se afecte positivamente los resultados de las pruebas saber pro.

Además, la lógica de privatización de la enseñanza superior, tanto por la creación de instituciones privadas como por las limitaciones presupuestarias impuestas a las universidades 
públicas, no apunta al desarrollo nacional de Ciencia y Tecnología.

En este contexto de limitaciones, se han evidenciado también, aunque dispersos, esfuerzos importantes. A finales de la década de los ' 80 s se inicia un movimiento para definir políticas de investigación en el país. En la década de los '90s, a partir de la promulgación de la Ley 29 de 1990, por la cual se dictan disposiciones para el fomento de la investigación científica y el desarrollo tecnológico, y se otorgan facultades extraordinarias, se va configurando el Sistema Nacional de Ciencia y Tecnología que prefigura la consolidación de COLCIENCIAS como Secretaría Técnica de Apoyo para la Investigación, y se promueve la discusión y el planteamiento de los diversos programas de investigación, como fueron: salud para la calidad de vida (del área de Salud); los retos de la diversidad (del área de ciencias humanas y sociales) y educación para la creatividad (del área de educación), entre otros.

Los programas planteados fueron revisados, actualizados y cualificados en su formulación y en su desarrollo, orientados a generar alternativas sistémicas relacionadas con la democratización de la Ciencia y la Tecnología, la regionalización del Sistema Nacional de Ciencia y Tecnología, la formación del potencial humano en todos los niveles y el fomento de grupos y centros de investigación en el cruce de las áreas de conocimiento y los desarrollos específicos de las instituciones.

Los logros alcanzados han sido variables y sometidos, entre otras cosas, a los vaivenes de asignación presupuestal en el nivel nacional, así como al centralismo y focalización de recursos. Frente a esto, el sistema en general y cada uno de los programas en particular, ha realizado los análisis respectivos y planteado las estrategias de ajuste. En la actualidad, el Sistema cuenta con ocho focos de investigación que involucran las áreas de Salud, Medio Ambiente, Agropecuaria, Educación, Biotecnología, Ciencias Básicas, Ciencias Sociales y Humanas, Industria, Energía, Minería y Mar. Cada uno de ellos define líneas de investigación, áreas prioritarias o estratégicas, $y$, de acuerdo con ellas, se hace análisis del contexto y de los problemas del conocimiento (Ministerio de Ciencia, Tecnología e Innovación, 2020).

\section{ASPECTOS DE LA INVESTIGACIÓN EN LAS IES.}

A pesar que, se ha reconocido un espectro importante de líneas de investigación, se sigue percibiendo una insularidad, sin una articulación orgánica, al interior de las Universidades en su conjunto frente a su contexto inmediato (sociedad y sector empresarial). Los programas académicos luchan por obtener sus registros calificados y por qué no, su acreditación de calidad, desconectados de lo que hacen otros programas de formación. No obstante, en los trabajos de grado, los cuales son realizados por los estudiantes, se constata una preocupación por ligarse a problemas del entorno. La participación de los docentes, sin embargo, es marginal, por cuanto se circunscribe a la asesoría puntual que demandan dichos trabajos.

En este orden de ideas, también se pudiera inferir que la marginalidad a la que se hizo referencia previamente es resultado de dos aspectos, primero, las cargas académicas derivadas de otras actividades que afectan el grado de profundidad con que el docente orienta las acciones propias de la investigación formativa y propone dinámicas para reconocer nuevos métodos y técnicas (Espinoza, 2020).

Por otra parte, el segundo aspecto se relaciona con la misma condición o estatus con el que el profesor aborda su saber investigativo, esto último, porque también se identifica que muchas acciones encaminadas a los fines formativos 
para la investigación son orientados por profesores que no cuentan con la experiencia (pese a contar con un estudio de posgrado) o al menos, no asumen esta labor como parte fundamental de su desempeño docente, lo cual, aunque pudiera considerarse una barrera fácil de superar, en la práctica pudiera existir desidia y rechazo por la complejidad y dedicación de tiempo que supone ahondar en la investigación y su rol transformador (Espinoza, 2020).

Desde una perspectiva adicional, el rol del docente $u$ orientador es clave en el desarrollo de las competencias que promueve la investigación formativa, por ello, el acompañamiento y la tutoría son esenciales para ofrecer una línea de argumentos que permitan al estudiante reconocer prácticas incorrectas y al tiempo, dotarlo de recursos de aprendizaje que le permitan superar el desconocimiento e ir caminando hacia la especialización de prácticas de investigación. Todo esto, se articula con una dedicación horaria suficiente para guiar, retroalimentar, proponer y ofrecer alternativas de solución a las deficiencias o vacíos identificados en un trabajo que resulta de un proceso de investigación formativa.

Igualmente, vale precisar que la cara opuesta de la postura mencionada con antelación es la del profesor que asume un rol de juez y limita su relacionamiento con el estudiante a la crítica no constructiva y la descalificación, posturas llamadas a ser relevadas con urgencia por otras que reconozcan la esencia de dar forma y las particularidades que esta implica.

De otra parte, no están definidos claramente el carácter y ámbito de los trabajos de la investigación formativa, y por tanto su pertinencia no es evaluable en términos del impacto en los problemas centrales de la sociedad.

Así mismo, la investigación no es tomada en cuenta en un momento de consolidación de las
IES como alternativa de formación en educación superior para la región, ni tampoco como factor generador de propuestas para la superación de los problemas sociales, ni incluso como factor de desarrollo de los saberes que cultiva y en los que forma. La investigación es un proceso tangencial dentro de la universidad, algo que se considera, pero sin comprometer a la institución como tal.

La inversión en investigación es mínima, pues las preocupaciones giran más en torno al desarrollo de la docencia calificada. Por lo mismo, la producción intelectual consiste en artículos publicados en las revistas indexadas, con una participación significativa de estudiantes, sobre todo de los últimos semestres de las carreras. Apoyo a lo que se llaman los semilleros de investigación, buscando una visibilidad de la investigación, tanto al interior de las IES como externamente, pero la interlocución con otras comunidades académicas de la región y del país todavía es reducida.

Tal como lo afirma Guerra, las universidades deben incentivar y propiciar la investigación, quizá fortaleciendo las convocatorias internas y con ellas el financiamiento de proyectos de investigación o estableciendo incentivos económicos como rebajas en las matriculas a los estudiantes que participen de grupos 0 semilleros de investigación, a los docentes para fortalecer su formación en investigación y la elaboración de artículos de alto impacto (2017, pág.88).

Sin embargo, en los últimos años, las exigencias del Ministerio de Educación Nacional (MEN) frente a la calidad de los programas académicos, han producido cambios cualitativos y cuantitativos. Se ha generado en las instituciones una mayor participación de los docentes, logrando conformar una masa crítica de investigadores, que ha sustentado académicamente a las Maestrías y Doctorados en las IES. Al mismo tiempo, se han construido 
derroteros metodológicos para los trabajos de grado, articulándose con la tipología de productos de MINCIENCIAS, lo cual ha redundado en un incremento del número de artículos elaborados por docentes y estudiantes, como resultado de procesos investigativos (González et al., 2020).

Se incrementaron las relaciones interinstitucionales con entidades nacionales e internacionales, a través de la realización de convenios. Se está respondiendo a las expectativas de la sociedad de una manera más coordinada, pero el desarrollo interno de la investigación aún sigue incipiente, por lo que puede decirse que, si bien se ha ganado en pertinencia, no se puede hablar de relevancia.

De esta manera se organizaron grupos de investigación, al interior de las universidades, organizados en Líneas de Investigación. Estos grupos se han conformado por profesores provenientes de diferentes disciplinas, de diferentes facultades $y$ relacionados con diversas unidades académicas. Todos buscando un propósito común: hacer investigación que favorezca la formación de los estudiantes y los mismos profesores. Cabe resaltar la vinculación de los Decanos y de los Coordinadores Académicos de todos los programas a esos grupos de investigación, como estrategia para fortalecer los procesos investigativos de la misma IES.

En esta vía, a partir de lo descrito por Gremmo y Gérard (2008), la investigación que se está generando desde las universidades podría pensarse en un proceso que podemos diseccionar por lo menos en tres elementos: 1) Se dota de herramientas al estudiante para investigar, siendo la enseñanza de métodos de investigación y teorías para comprender los fenómenos sociales y naturales la forma como se cimentan las bases de producción intelectual a diversos niveles; 2) Existe siempre un investigador, en muchos casos profesores de la institución, que puede transmitir a los estudiantes estas herramientas para la investigación e innovación; 3) finalmente, existe un proceso de autoaprendizaje por parte del estudiante en los diferentes escenarios de investigación formativa: "semilleros" de investigación, trabajos de grado, entre otros espacios.

Conceptualmente y en integración con los argumentos hasta ahora mencionados, en la literatura se identifican múltiples formas para caracterizar las dimensiones que hacen parte de categoría investigación formativa, por ejemplo, Tanda et al., (2020) agotan el constructo en nueve dimensiones, estos son: (1) la problematización de la situación en cuestión, (2) las diferentes representaciones, (3) la actualización o producción de conocimientos y la modificación de relación con el conocimiento así como con el mundo, (4) el desarrollo de una cultura científica, (5) el desarrollo de proceso reflexivo, (6) transformación de identidad, (7) autoformación, (8) escritura como herramienta de profesionalización, y, (9) la integración de varias dimensiones.

Desde otra perspectiva, para autores como López et al. (2017) y Rojas y Aguirre (2015), la investigación formativa es vista desde dos concepciones, lo pedagógico y el desarrollo de competencias investigativas, por ello, sus constructos se sintetizan en tres elementos: (1) conocer-hacer (relacionado con un ejercicio idóneo, estructurado y proactivo); (2) saberhacer (remite a la aplicación de habilidades y competencias investigativas) y (3) saber-ser (actitudes y manifestaciones de la personalidad tendientes a profundizar en el proceso investigativo).

En este sentido, se podría pensar la investigación formativa como un espacio de aprendizaje de métodos y teorías que es guiada por un profesor o investigador consagrado que transmite su conocimiento, a la vez que el estudiante genera 
mecanismos de autoaprendizaje con los cuales puede profundizar o criticar los conocimientos adquiridos en la institución de educación superior a la que pertenece.

Así pues, la investigación formativa tiene por actores fundamentales al estudiante $y$ al docente, a la vez que se ven enmarcados en códigos, normas y saber hacer institucionales. Por lo anterior, estudiar a la investigación formativa desde una perspectiva integral exige el abordaje de los sentidos y representaciones que sobre esta aportan sus diversos actuantes: estudiantes, profesores, instituciones de educación superior, organismos estatales, agremiaciones, entre otros (Turpo et al., 2020).

Ahora bien, el docente transmite conocimientos para la investigación, pero ¿cómo lo hace? Para pensar en una respuesta a esta pregunta, se podría pensar en la transmisión del oficio. Como afirma Pierre Bourdieu (2002), el conocimiento en investigación de cualquier ciencia u oficio se transmite del profesor al estudiante como quien enseña un oficio en un taller de ebanistería. Es realizando investigación entre ambos actores que quien está aprendiendo logra interiorizar los elementos asociados a esta actividad. En este sentido, la investigación formativa parte de la relación profesor - estudiante, en el que el segundo está constantemente absorbiendo conocimiento del primero.

Además de la relación entre ambos actores del proceso de investigación formativa, también encontramos la constitución de comunidades de práctica ancladas a las actividades de investigación propiamente dichas. Según Wenger y Snyder (2000), las comunidades de prácticas son una nueva forma de organización social en la que se genera un impulso del conocimiento, su intercambio entre los investigadores y el aprendizaje en este proceso.

Así, estos grupos son un conjunto de profesionales o practicantes que por intereses comunes generan conocimiento en común (Barragán, 2015). De tal manera, más allá de los semilleros de investigación formativa que existen en cada una de las instituciones de educación superior, se puede constatar diversas redes de generación y transferencia de conocimiento que fortalecen la producción en investigación y las capacidades de los estudiantes en esta materia.

Profundizando en la idea de la investigación formativa antes expuesta, valdría pluralizar que esta se agota en varios ambientes al interior de las instituciones de educación superior, tal como son los que hacen parte de los contenidos curriculares (cursos, trabajo de grado, otros) y otros que por su naturaleza escapan al currículo y se configuran en ambientes alternos de aprendizaje sobre la investigación, como los semilleros.

\section{REFLEXIONES Y RECOMENDACIONES SOBRE LA INVESTIGACIÓN FORMATIVA}

Por tanto, se deben abrir los escenarios para que se puedan enfocar en la investigación propiamente dicha.

$\otimes \quad$ La diversidad en los sentidos y subjetividades con respecto a la investigación formativa por parte de profesores, si bien en primera instancia no remitiría explícitamente a una problemática, si agudiza esta última situación en el momento en que la categoría conceptual se torna imprecisa, confusa y desconocida, dado que, las acciones concretas de sus actuantes pudieran tornarse desarticuladas a sus propósitos primarios (Turpo, 2020).

\ Retomando la anterior mención, es relevante anotar que las prácticas del profesor configuran, pedagógica y didácticamente, el pilar que soporta el proceso de enseñanza a "investigar investigando", por lo tanto, es pertinente la construcción de un imaginario 
que dirija las acciones formativas a las especificidades que requiere el desarrollo de las capacidades investigativas en los estudiantes, hecho que solo será posible con la articulación de un repensar por parte de los profesores sobre sus recursos y métodos, al tiempo que desde las instituciones de educación o de la media académica (para el caso colombiano), se le brindan los espacios y condiciones para planear el liderazgo de acciones tendientes a la investigación formativa (Rojas y Aguirre, 2015; Iriarte, 2020).

\ Preponderancia en la formación disciplinar específica de cada cuerpo de conocimiento segmentado en las IES por programas académicos y facultades, reduciendo significativamente la oportunidad de abrir espacios para procesos como, los semilleros de investigación, en el campo de la investigación formativa (Molina y Fonseca, 2019).

\section{REFERENCIAS BIBLIOGRÁFICAS}

1. Banco Mundial (2018). Informe sobre el desarrollo mundial 2018: Aprender para hacer realidad la promesa de la educación, cuadernillo del "Panorama general", Banco Mundial, Washington, DC. Licencia: Creative Commons de Reconocimiento CC BY 3.0 IGO.http://iin.oea.org/pdf-iin/RH/ docs-interes/2019/Informe-sobre-el Desarrollo-Mundial-2018.pdf

2. Barragán, D. F. (2015). Las Comunidades de Práctica (CP): hacia una reconfiguración hermenéutica. Franciscanum. Revista de las ciencias del espíritu, LVII (163), pp. 155 - 176.

https://www.redalyc.org/articulo. oa?id=343533885005.

3. Bourdieu, Pierre (2002). El oficio del sociólogo. Siglo XXI Editores.
4. Comisión Económica para América Latina y el Caribe (CEPAL). (2020). Panorama Fiscal de América Latina y el Caribe 2020. Santiago: CEPAL. Págs. 9-48.

5. Correa D., A. M., Benjumea A., M., \& Valencia A., A. (2019). La gestión del Conocimiento: una alternativa para la solución de problemas educacionales. Revista Educare.

6. Espinoza, E. E. (2020). La investigación formativa. Una reflexión teórica. Revista Conrado, 16 (74), 45-53. http://scielo. sld.cu/pdf/rc/v16n74/1990-8644rc-16-74-45.pdf

7. González, C. H., Murillo, G y Urrego, D. (2020) Transformación del Sistema de Aseguramiento de la Calidad de la Educación Superior en Colombia 20192020. Revista Educación y Humanismo. Vol. 22 no. 38 https://doi.org/10.17081/ eduhum.22.38.3541

8. Guerra M. René A. (2017). ¿Formación para la investigación o investigación formativa? La investigación y la formación como pilar común de desarrollo. Revista Boletín Redipe. Vol. 6. Núm. 1

9. Iriarte, A. (2020). Fenomenologíahermenéutica de la investigación formativa. El formador de formadores: de la imposición a la transformación. Revista de investigación, desarrollo e innovación, vol. 10 (2), pp. 311-322. https://doi.org/10.19053/20278306.v10. $\underline{\mathrm{n} 2.2020 .10722}$ 
10. López-de parra, L., Polanco, V y Correa, L. (2017). Mirada de las investigaciones sobre formación investigativa en la universidad latinoamericana: estado del arte (2010- 2017). Revista de Investigación, Desarrollo e Innovación, vol. 8, n. 1, p. 77-95.

https://doi.org/10.19053/20278306. v8.n1.2017.7371

11. Gremmo, M y Gérard, L. (2008). «Accompagner les apprentischercheurs jeux et enjeux de la direction de mémoire», Recherche et formation [En línea], 59 | 2008. http://journals. openedition.org/rechercheformation/621

12. Martínez, L. R y Beleño, Y. (2020). Las políticas implementadas para mejorar la calidad educativa en la educación superior de Colombia. Formación Educativa en el Contexto Social $y$ Cultural. Vol 4. Universidad de Zulia. Pág. 251 - 264.

\section{https://www.corposucre.edu.co/sites/} default/files/investigacion/Libro\%20 UNIZULIA $\% 202020 \% 20$ Contexto $\% 20$ social\%20educativa\%20Vol\%20IV.pdf

13. Ministerio de Ciencia, Tecnología e Innovación. (2020). Descripción de Focos y líneas temáticas. Anexo 1. Convocatoria para el fortalecimiento de CTel en instituciones de educación superior (IES) públicas 2020

https://minciencias.gov.co/convocatorias/ programa-y-proyectos-ctei/ convocatoria-para-el-fortalecimientoctei-en-instituciones
14. Molina, Y. A y Fonseca, J. E. (2019). La investigación formativa contable en universidades de Tunja. Revista de investigación, desarrollo e innovación RIDI., vol. 10 (1), pp. 93106. https://revistas.uptc.edu.co/index. php/investigacion_duitama/article/ view/10014

a.

15. Pardo, C. (2019). El reto de invertir en ciencia, tecnología e innovación en Colombia. Universidad del Rosario. https://www.portafolio.co/economia/elreto-de-invertir-en-ciencia-tecnologia-einnovacion-en-colombia-529537

16. Rodríguez, J. A y Torres, D. A. (2020) Colombia construye sus cimientos. Propuesta de foco de ciencias básicas y del Espacio. Volumen 4. Universidad Nacional. https://minciencias.gov.co/ sites/default/files/upload/paginas/mision_de_sabios_ciencias_7-9-20doc. pdf

17. Rojas, C y Aguirre, S. (2015). La formación investigativa en la educación superior en América Latina y el Caribe: una aproximación a su estado del arte. Revista Eleuthera, n. 12, pp. 197-222. 10.17151/eleu.2015.12.11

18. Serbin, A. (2018). América Latina y el Caribe frente a un nuevo orden mundial: poder, globalización y respuestas regionales. Buenos Aires: Icaria. Págs. 46-51 
19. Turpo, O., Quispe, P, M., Paz, L. C y Gonzáles, M. M. (2020). La investigación formativa en la universidad: sentidos asignados por el profesorado de una Facultad de Educación. Educ. Pesqui. Vol.46. https://doi.org/10.1590/s16784634202046215876.

20. UNESCO -IESALC y Universidad Nacional de Cordoba. (2018). El papel estratégico de la Educación Superior en el desarrollo sostenible de América Latina y el Caribe. Córdoba: Universidad Nacional de Córdoba. Págs. 213-215.

21. Wenger, E y Snyder, W. (2000) «Communities of Practice: the Organizational Frontier», Harvard Business Review 78, Vol. 1: 139-145. https://hbr.org/2000/01/communities-ofpractice-the-organizational-frontier 